\title{
Talking about space: The role of parent and child sex on the frequency of parental spatial talk, and its association with child numeracy and school readiness
}

Elian Fink, Claire Hughes, Henriette Hendriks, Emily K. Farran \& Katie Gilligan-Lee

\section{Previous Research}

Parental talk plays an important role in facilitating children's cognitive development and academic success. In addition to the broad role of overall linguistic input, there has been a focus on finegrained aspects of parental speech and corresponding aspects of child development. One such aspect is parental spatial language which has been shown to independently facilitate children's spatial abilities (e.g., Gunderson \& Levine, 2011; Pruden et al., 2011).

\section{Method}

\section{Participants}

66 families (42 families with sons) were recruited from infancy from Cambridge, UK and were seen at home when children were 24 months of age, and at the university lab at 48 months of age.

\section{Measures}

Parental Spatial Talk at 24 and $\mathbf{4 8}$ months Spatial terms were coded during parent-child block play, specifically:

- spatial dimensions (e.g., big, small)

- shapes (e.g., square)

- locations and directions (e.g., up),

- orientations and transformations (e.g., around),

- continuous amount (e.g., some),

- deictic terms (e.g., here),

- spatial features and properties (e.g., side),

- pattern (e.g., sequence)

\section{Parental Verbal Ability}

Mothers and fathers completed the National Adult

Reading Test (Nelson \& Wilson, 1991)

\section{Child Numeracy at $\mathbf{4 8}$ months}

24-item test of numerical ability (PEN-B) for children between 3 and 6 years of age (Purpura et al., 2015)

Child School Readiness at $\mathbf{4 8}$ months of age Nursery teachers rated children's language and cognition using the BESSI (Hughes et al., 2015)
Importantly, child's spatial abilities predict later achievement in areas of STEM (Wai, Lubinski, \& Benbow, 2009), where a gender gap exists, with males more likely to pursue these fields of study compared to females (Cimpian et al., 2020). Differences in parental input of early spatial talk to boys compared to girls has been put forward to partially explain this gap. Indeed, research has shown that boys tend to hear more spatial words from their parents compared to girls (e.g., Pruden \& Levine, 2017). However, the role of father's spatial talk has been overlooked in this field, with studies often focusing exclusively on mothers.

\section{The Current Study}

In this study we asked:

(1) Do mothers and fathers vary in their spatial talk to children?

(2) What is the interplay between parent and child sex with respect to parental spatial talk?

(3) Is the role of parental spatial talk for child outcomes the same for mothers and fathers?

\section{Results}

\section{Do mothers and fathers vary in their spatial talk to children?}

There was no difference in the amount of mother $(\mathrm{M}=3.33, \mathrm{SD}=3.48)$ and father $(2.91, \mathrm{SD}=3.49)$ spatial talk, $t=.78$, $p>0.05)$ directed at their child at 24 months, or between mother $(M=7.73, S D=3.09)$ and father $(7.95, S D=2.66)$ spatial talk, $t=-.43, p>0.05$, 48 months.

2. What is the interplay between parent and child sex with respect to spatial talk?

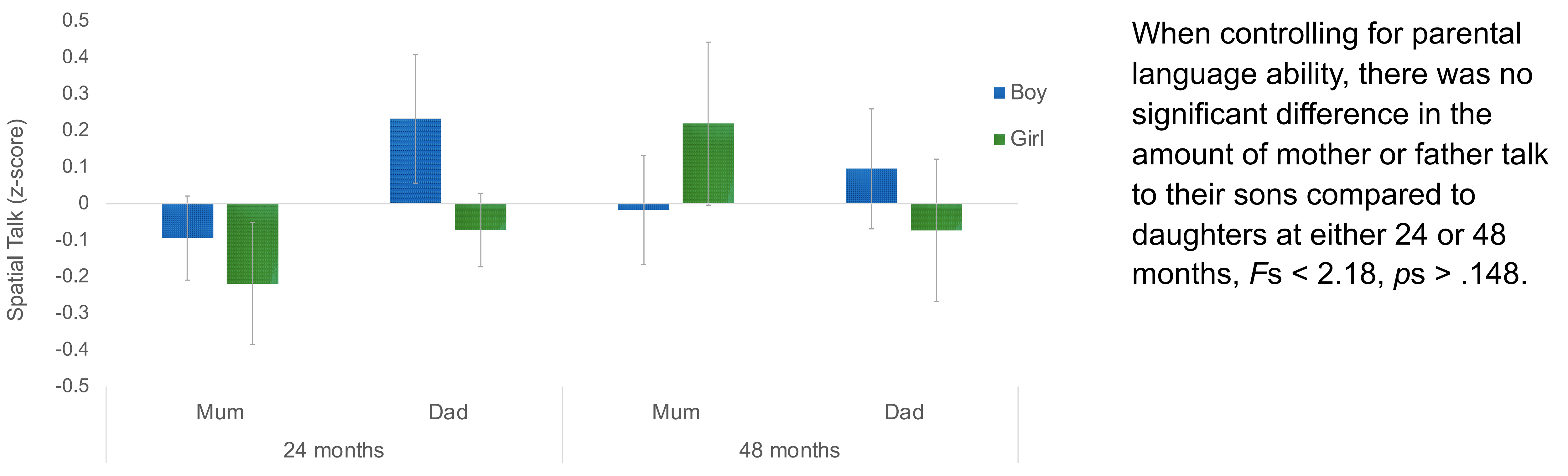

3. Is the role of spatial talk for children's school readiness the same for mothers and fathers?

When comparing the association between maternal and paternal spatial talk and child outcomes (numeracy and school readiness), only paternal spatial talk was significantly associated with spatial talk, $r=.45, p=.005$, even when controlling for parental language ability.

\section{Conclusions}

This is the first study to our knowledge to directly compare the amount of spatial talk to children from mothers and fathers longitudinally, and examine whether the salience of spatial talk for child outcomes varies by parent sex.

The current findings showing no difference between mother and father spatial talk to sons and daughters is somewhat surprising and final coding of the further 60 that took part in the current study will help clarify this result.
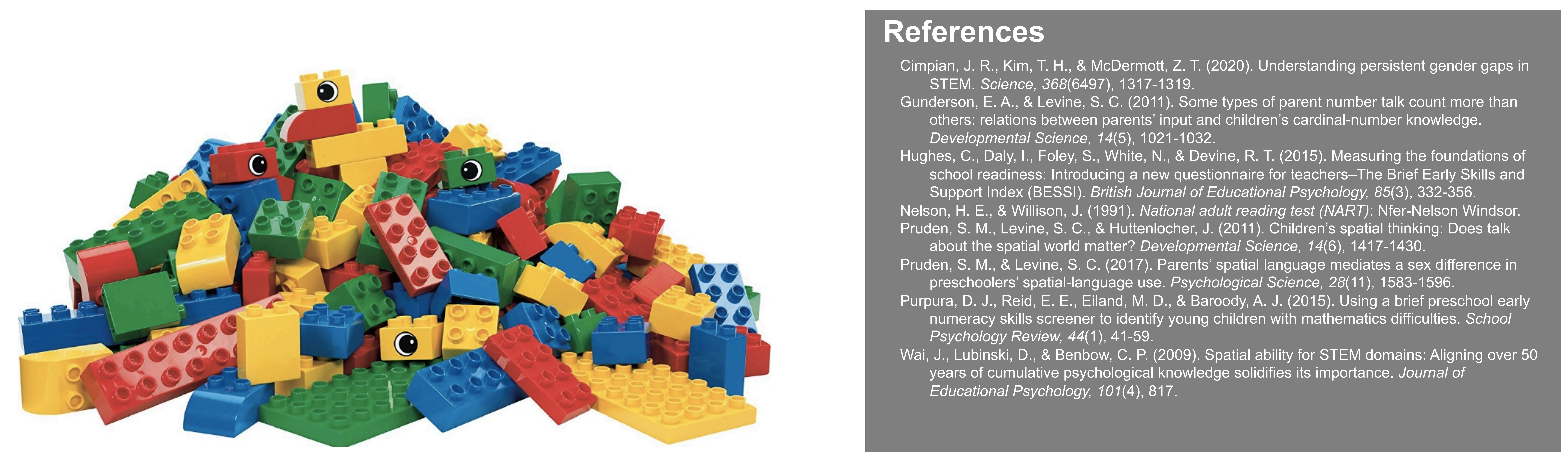